\title{
The TRPV2 cation channels: from urothelial cancer invasiveness to glioblastoma multiforme interactome signature
}

\author{
Giorgio Santoni ${ }^{1}$ - Consuelo Amantini ${ }^{2} \cdot$ Federica Maggi $^{1,3} \cdot$ Oliviero Marinelli $^{1,2} \cdot$ Matteo Santoni $^{4}$. \\ Massimo Nabissi $i^{1} \cdot$ Maria Beatrice Morelli $^{1}$
}

Received: 3 July 2019 / Revised: 5 September 2019 / Accepted: 6 September 2019 / Published online: 25 October 2019

(c) The Author(s), under exclusive licence to United States and Canadian Academy of Pathology 2019

\begin{abstract}
Changes in transient receptor potential (TRP) $\mathrm{Ca}^{2+}$ permeable channels are associated with development and progression of different types of cancer. Herein, we report data relative to the expression and function of TRP vanilloid 2 (TRPV2) channels in cancer. Overexpression of TRPV2 is observed in high-grade urothelial cancers and treatment with the TRPV2 agonist cannabidiol induces apoptosis. In prostate cancer, TRPV2 promotes migration and invasion, and TRPV2 overexpression characterizes the castration-resistant phenotype. In breast cancer cells, inhibition of TRPV2 by tranilast reduces the insulinlike growth factor-1 stimulated proliferation. TRPV2 overexpression in triple-negative breast cancer cells is associated with high recurrence-free survival. Increased TRPV2 overexpression is present in patients with esophageal squamous cell carcinoma associated with advanced disease, lymph node metastasis, and poor prognosis. Increased TRPV2 transcripts have been found both in benign hepatoma and in hepatocarcinomas, where TRPV2 expression is associated with portal vein invasion and reduction of cancer stem cell expression. TRPV2 expression and function has been also evaluated in gliomagenesis. This receptor negatively controls survival, proliferation, and resistance to CD95- or BCNU-induced apoptosis. In glioblastoma stem cells, TRPV2 activation promotes differentiation and inhibits the proliferation in vitro and in vivo. In glioblastoma, the TRPV2 is part of an interactome-based signature complex, which is negatively associated with survival, and it is expressed in high risk of recurrence and temozolomide-resistant patients. Finally, also in hematological malignancies, such as myeloma or acute myeloid leukemia, TRPV2 might represent a target for novel therapeutic approaches. Overall, these findings demonstrate that TRPV2 exhibits an oncogenic activity in different types of cancers, controlling survival, proliferation, migration, angiogenesis, and invasion signaling pathways. Thus, it prompts the pharmacological use of TRPV2 targeting in the control of cancer progression.
\end{abstract}

These authors contributed equally: Giorgio Santoni, Consuelo Amantini

$\triangle$ Giorgio Santoni

giorgio.santoni@unicam.it

1 School of Pharmacy, University of Camerino, via Madonna delle Carceri 9, 62032 Camerino, MC, Italy

2 School of Biosciences and Veterinary Medicine, University of Camerino, via Madonna delle Carceri 9, 62032 Camerino, MC, Italy

3 Department of Molecular Medicine, Sapienza University, Viale Regina Elena 324, 00161 Rome, RM, Italy

4 Medical Oncology Unit, Hospital of Macerata, Via Santa Lucia 2, 62100 Macerata, MC, Italy

\section{Introduction}

Numerous recent studies demonstrate the role of ion channels in the pathogenesis of several chronic inflammatory disease, hence the term "channelopathies". At present, in mammalian cells, 28 transient receptor potential (TRP) $\mathrm{Ca}^{2+}$-permeable channels, belonging to six subfamilies (TRPV, TRPM, TRPC, TRPA, TRPP, and TRPML) and activated by chemical and sensory agonists, have been reported [1]. The vanilloid subfamily (TRPV) is represented by six members (TRPV1-6). TRPV1 is the prototype of TRPV channels, it is activated by heat and synthetic or endogenous vanilloids [2]. TRPV2 shares about 50\% sequence identity with TRPV1 $[3,4]$. Nevertheless, TRPV2 shows distinct cellular functions from those mediated by TRPV1 [5]. TRPV1 expression is mainly localized in the plasma membrane [3, 4]; in contrast, TRPV2 is localized in the intracellular membranes [6]. 
Moreover, TRPV2 is not stimulated by heat or in vivo by vanilloid exposure [3, 5-7]. In dorsal root ganglion neurons, TRPV2 showed a punctate distribution and $\mathrm{Rab} 7$ endosomal co-localization, where it promotes neuronal outgrowth and signaling [5]. TRPV2 activity is induced by 2-aminoethoxydiphenyl borate (2-APB), probenecid and cannabidiol (CBD), and inhibited by Ruthenium red (RR), Gadolinium (Gd), and tranilast [2, 4, 8-10]. Physiologically, post transcriptional modifications, lipid- and protein-protein interactions are involved in TRP regulation [1]. Phosphatidylinositol 4,5-bisphosphate (PIP2) and phosphorylation, due to extracellular signal-regulated kinase (ERKs), regulates the TRPV2 functions [5, 11].

For information regarding the structure of TRPV2, we refer the readers to the very informative and innovative works in which the TRPV2 full-length structure is described by cryo-electron microscopy [12], the conformational plasticity [13] and the distinct TRPV2 conformational states [14].

It is now clear that TRPV2, expressed not only in sensory neurons but also in motor neurons and in many nonneuronal tissues, displays tissue-specific physiological functions [15]. It is involved in perception of noxious stimuli, due to its stretch-dependent properties, and in the regulation of calcium homeostasis functioning as a mechanosensor, thermosensor, and lipid sensor.

TRPV2-mediated signaling pathways have profound effects on a variety of pathological processes such as cardiomyopathies [16, 17] and muscular dystrophy [18, 19], diabetes and obesity [20, 21], fibromyalgia [22, 23], infection diseases and so forth [24, 25], and cancer.

The aim of this review is to report the involvement of TRPV2 channels in the development, progression, and metastatic organ invasion in different tumor types. The stimulation of TRPV2 by growth factors, cytokines, hormones, and endocannabinoids induces the translocation of TRPV2 from the endosome to the plasma membrane, affecting both cell proliferation, and cell death [26]. Loss or changes of TRPV2-mediated signals result in uncontrolled proliferation and apoptotic-resistance, whereas TRPV2 activation enhances the migration and increases the invasiveness of cancer cells [27]. Finally, alternative splice variants of TRPV2 mRNA, evidenced in cancer cells, inhibit the TRPV2 trafficking/translocation [28].

In conclusion, the regulation of TRPV2 channel expression could control tumor progression and therefore these channels represent novel targets for diagnosis and therapy.

\section{TRPV2 expression in urogenital cancer types}

The history of the expression and functions of the TRPV2 channel in cancer cells begin in 2008 in the Santoni laboratory whereby a study from Caprodossi et al. demonstrated that
TRPV2 expression in normal human urothelial tissues and its increased levels in high-grade and -stage urothelial cancer (UC) tissues and cell lines [29]. Both the full-length TRPV2 (f-TRPV2) and the short splice variant (s-TRPV2) are expressed in normal human urothelial cells and in normal bladder tissues; however, a progressive reduction of TRPV2 splice variant is detected from low stages (e.g., pTa, pT1, and pT2) to the high-stage (pT3 and pT4). In addition, a significant increase of full-length TRPV2 in high-grade and high-stage UC has been reported [29]. Subsequently, a functional role of the TRPV2 was demonstrated by Shimada and collaborators in the bladder cancer (BC) cell line T24 treated with CBD [30]. TRPV2 activation increases the intracellular calcium levels, decreases cell viability and induces apoptotic cell death [30]. Furthermore, the contribution of TRPV2 in matrix metalloproteinase-2 (MMP2)-mediated migration and invasion was demonstrated by Liu and Wang in the human 5637BC cell line transfected with rat TRPV2 [31]. Mizuno et al. reported higher TRPV2 expression levels in the murine MBT-2 BC cell line compared with normal mouse urothelial cells; the silencing of TRPV2 by RNA interference in MBT-2 cells increased cell proliferation, whereas the opposite effect was obtained by using TRPV2 activators. In addition, dominant-negative TRPV2 channel reduced $\mathrm{Ca}^{2+}$ permeability in MBT-2 cells, compared with controls [32].

A role for TRPV2 in cancer migration was further demonstrated in PC3 cell line by Prevarskaya's group [33]. It was reported that lysophospholipids (e.g., lysophosphatidylcholine and lysophosphatidylinositol) induce a TRPV2-mediated calcium influx, via Gq/Go and phosphatidylinositol-3,4 kinase (PI3,4K) signaling and translocation of TRPV2 from endosome to the plasma membrane, leading to increased PC3 prostate cancer (PCa) cell migration [33]. Moreover, the TRPV2 mRNA levels were higher in metastatic cancer patients compared with non metastatic primary tumors, with the higher TRPV2 expression characterizing the castration-resistant PCa phenotype. TRPV2 transfection in $\mathrm{LNCaP}$ prostate cell line increased cell migration and the expression of MMP-9 and cathepsin B [33]. Similarly, in a nude mice xenograft, TRPV2 silencing reduced PC3 cell growth and invasion, prompting TRPV2 as prognostic and therapeutic target in advanced PCa [34]. A recent publication reported that during cancer invasion TRPV2 activation and clustering by focal mechanical stimulation increases motility and causes actin reorganization that fuel the metastasis spreading [35].

Recent data show a role for adrenomedullin (AM), calcitonin (CT), and calcitonin gene-related peptide in PCa and bone metastasis. AM has been reported to induce a TRPV2dependent prostate and $\mathrm{BC}$ cell migration and invasion; it affected the receptor activator of nuclear factor kappa-B ligand (RANKL) levels in the bone, favoring the metastasis formation [36, 37]. Moreover, CT acts on protein kinase-A 
to promote PCa metastasis. Deletion of the PDZ-binding domain of CT receptor abrogates the bone metastasis in an orthotopic PCa model [37]. TRPV2 is also a positive modulator of tumor endothelial cell proliferation and, together with TRPC3 and TRPA1, represents an overexpressed prostate-associated gene in PCa [38].

Overall, TRPV2 activation promotes PI3,4K- and protein kinase A-dependent migration and metastasis in PCa cells, respectively, and through continuous $\mathrm{Ca}^{2+}$ influx, inhibits cell proliferation and induces apoptosis of BC cells. Altered transcription of TRPV2 generates a s-TRPV2 splice variant whose levels decrease during UC progression. Although the pathophysiological relevance of the splicing process is still not well understood in BCs, overexpression of s-TRPV2 demonstrated in leukemic blast cells (LBCs) was found to impair the f-TRPV2 translocation to plasma membrane and to inhibit its $\mathrm{Ca}^{2+}$ activity [39]. Thus, the ability of tranilast to restore the s-TRPV2 expression levels in LBCs [39], strongly suggest the possible usage of TRPV2 antagonist also in advanced UC.

\section{Expression and function of TRPV2 in breast cancer cells}

Breast cancer is the most frequent cancer type in women, with a high mortality rate in metastatic patients. It has been demonstrated that the TRPV2 antagonist, tranilast, reduces the IGF 1-induced calcium rise and cell proliferation in MCF-7 cell line by inducing hypo-phophosphorylation of the retinoblastoma protein and $\mathrm{G} 1$ arrest [40, 41]. Moreover, reduced proliferation, promoted by the TRPV2 agonist CBD through a sustained upregulation of ERK activity, has been reported in aggressive breast cancer cells [42]. In addition, it has been shown that TRPV2 activation promotes cell differentiation in glioblastoma (GBM) stem cells [43] and reduces colony formation in liver cancer stem cells (CSC) [44] suggesting other TRPV2-mediated signals in stem cells-derived cancers. Thus, differences in TRPV2signaling pathway activation may be responsible for paradoxical effects of the TRPV2-mediated functions.

TRPV2 has been also found to bind and inhibit Rac1 and RhoA in fibroblast-like synoviocytes from rheumatoid arthritis patients [45]. Concordantly in breast cancer cells, a reduction of TRPV2 levels by treatment with two Rhokinase inhibitors, fasudil, and Y-27632, has been demonstrated in ZR-75-1, MCF-7, and MDA-MB-231 BC cell lines [46]. In addition, treatment of human breast cancer cell lines with the antimicrobial peptide hCAP18/LL37 stimulated migration by recruiting the TRPV2 channel to pseudopodia through PI3K/Akt activation (Fig. 1). $\mathrm{Ca}^{2+}$ entry synergizes with a $\mathrm{K}^{+}$efflux through the bradykinin channel, BKCa. No major differences were observed by using the D- or L-enantiomer of LL-37 suggesting a TRPV2-independent decrease membrane fluidity [47].

Triple-negative breast cancer (TNBC) is highly aggressive and difficult to treat. Recent studies have demonstrated upregulation of the TRPV2 protein in TNBC tissues compared with normal tissues. Moreover, a direct correlation between TRPV 2 overexpression and higher recurrence-free
Fig. 1 Mechanism proposed for the stimulatory activity of the LL-37 antimicrobial peptides on cell migration. LL-37 binds to the membranes of caveolae and pseudopodia [1], and activates $\mathrm{PI} 3 \mathrm{~K} / \mathrm{AKT}$ signaling [2]. AKT induces the recruitment of the TRPV2 channel [3] from intracellular vesicles to plasma membranes of pseudopodia. The increase of intracellular $\mathrm{Ca}^{2+}$ induced by TRPV2 is accompanied by $\mathrm{K}^{+}$efflux through BKCa [4], which preserves the ion balance and maintain the $\mathrm{Ca}^{2+}$ entry, which promotes cancer cell migration. Courtesy by Gambade et al. [47]

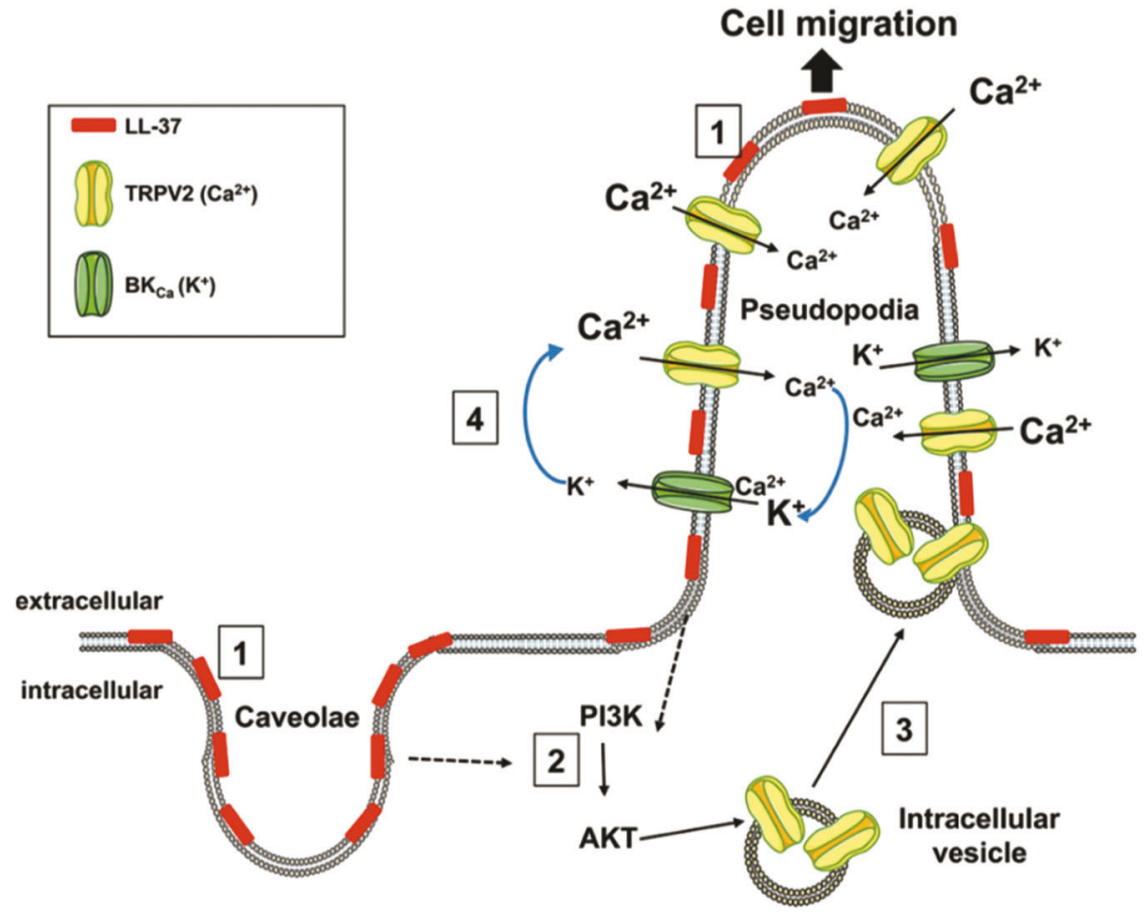


survival, was seen in TNBC and estrogen receptor $\beta$ (ER $\beta)$ negative breast cancer patients who underwent a chemotherapy regimen [48] (Fig. 2). TRPV2 overexpression or CBD treatment enhanced doxorubicin (DOXO) uptake and apoptotic cell death in TNBC cells. These effects were reverted by TRPV2 blocking or downregulation [48]. In an

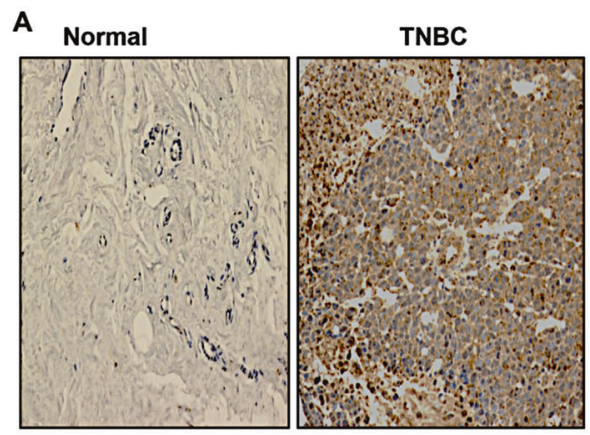

C

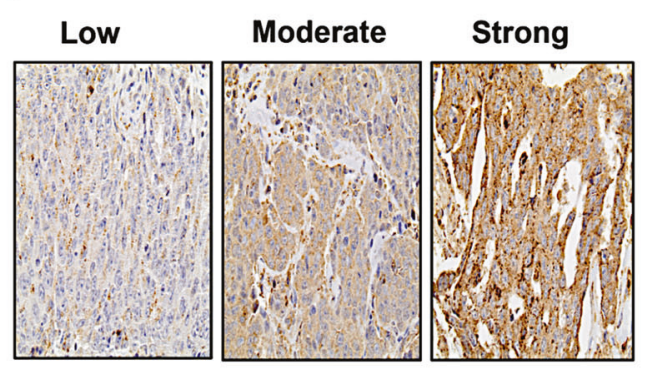

in vivo mouse model, increased apoptosis was observed in TNBC tumors from CBD plus DOX-treated mice, compared with mice treated with CBD or DOXO alone. Thus, these studies identify a good prognostic role for TRPV2 in TNBC and ER $\beta$-negative BC patients, and suggest that TRPV2 activation may represent a new therapeutic strategy
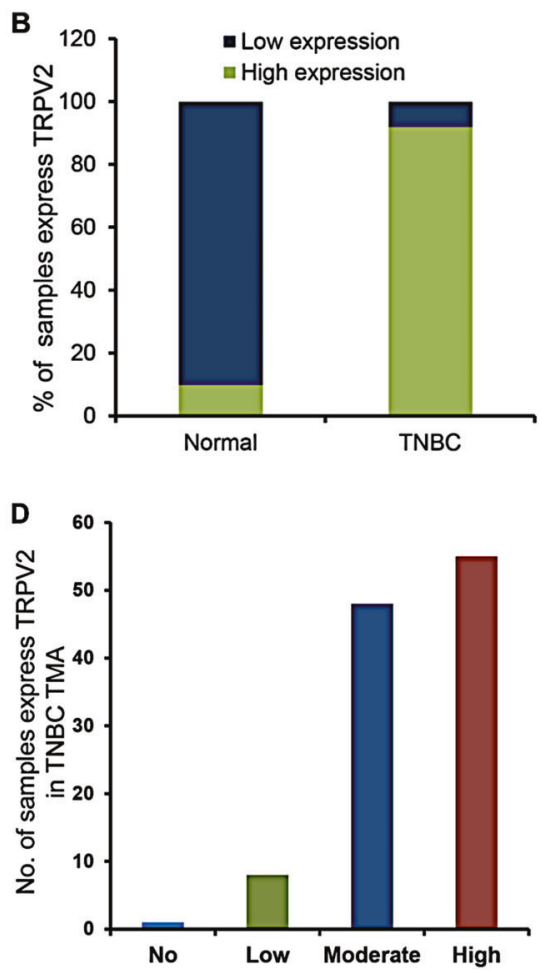

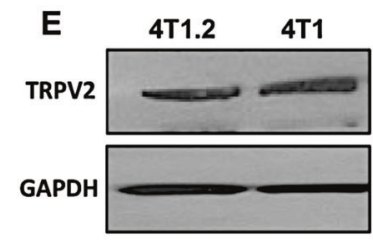

SUM MDAMB231 SCP2
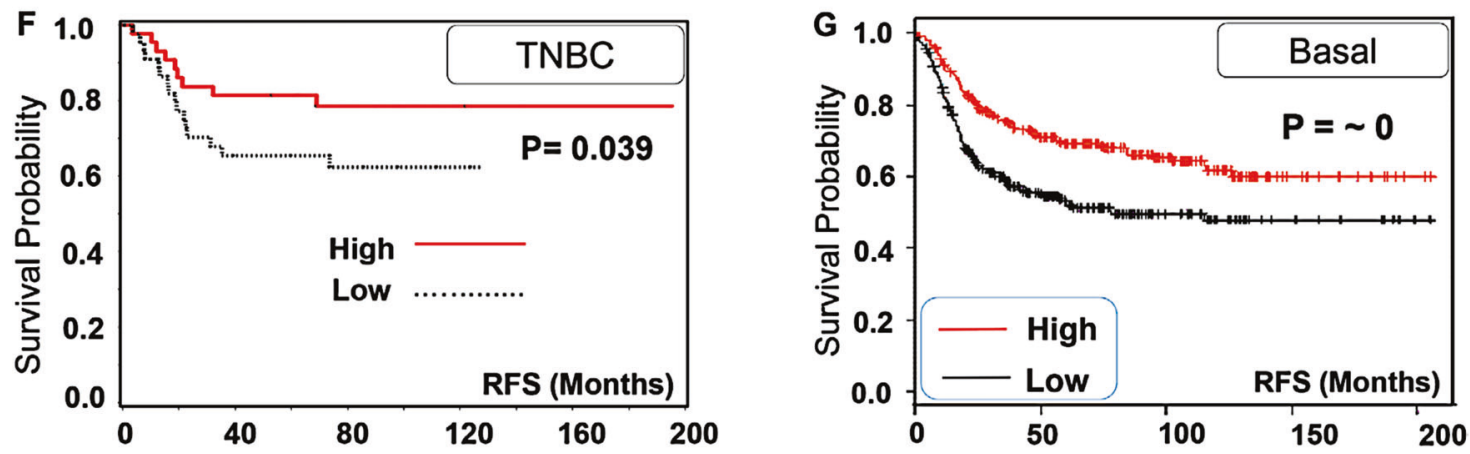

Fig. 2 TRPV2 is highly expressed in TNBC tissues and associated with better prognosis. a Representative image of immunehistochemical staining showing TRPV2 expression in normal and TNBC breast tissues. b Quantitation of TRPV2 expression of normal and TNBC breast tissues. c Representative image of IHC TRPV2 staining in the tissue microarray of TNBC patient samples. d Quantitation of TRPV2 expression in the tissue microarray of TNBC

patients. e Western blot image showing TRPV2 protein expression of 4T1.2, 4T1, SUM159, MDA-MB231, and SCP2 cells. GAPDH used as a loading control. f Tissue microarray analysis showing the recurrence-free survival (RFS) of TNBC patients $(n=116)$ of high/ low TRPV2 protein expression. g Kaplan-Meier blot showing RFS of high/low expressing TRPV2 breast cancer patients of basal subtypes ( $n=580$ patients). Courtesy of Elbaz et al. [48] 
to increase the chemotherapic effects in these $\mathrm{BC}$ patients [48] (Fig. 3).

Overall, overexpression of TRPV2 in TNBC and ER $\beta$ negative breast cancer plays a protective role by increasing the sensitivity of breast cancer cells to chemotherapyinduced apoptosis representing a positive prognostic factor. The increase of TRPV2 expression by CBD treatment, that sustains the ERK-dependent inhibition of breast cancer proliferation, or genetic/pharmacological downregulation of TRPV2 which reduces apoptosis, chemoresistance, and migration, may together represent a new therapeutic strategy for care this aggressive cancer.

\section{Role of TRPV2 in in esophageal squamous cell carcinoma (ESCC)}

TRPV2 mRNA overexpression was detected in ESCC tissues and ESCC cell lines respect to normal cells [49]. Moreover, recently, Huang et al. confirmed that TRPV2, but also TRPV1 and TRPV4, are functional and upregulated in ESCC. The TRPV2 channel was activated by exposure of ESCCs to high temperatures $\left(53^{\circ} \mathrm{C}\right)$ or to a newly developed cannabinoid, O1821 [50].

High TRPV2 expression was observed in patients with advanced disease, high-stage and lymph node metastasis. The 5-year disease-specific survival and disease-free survival were found to be worse in patients harboring high TRPV2 level compared with those showing low TRPV2 expression [49]. By multivariate analysis the TRPV2 mRNA expression and lymph node invasion were independent prognostic factors. TRPV2 mRNA overexpression represents a negative prognostic factor in ESCC patients surgical resected [49]. Moreover, CSC isolated from TE8 ESCC cell line also showed TRPV2 upregulation. CSCs were more sensitive than non-CSCs to tranilast effects that decreased the tumor sphere numbers. Furthermore, in TE8 cell line, tranilast reduced the stemness potential, by inhibiting the aldehyde dehydrogenase 1-positive CSCs, suggesting that TRPV2 might be involved in CSC
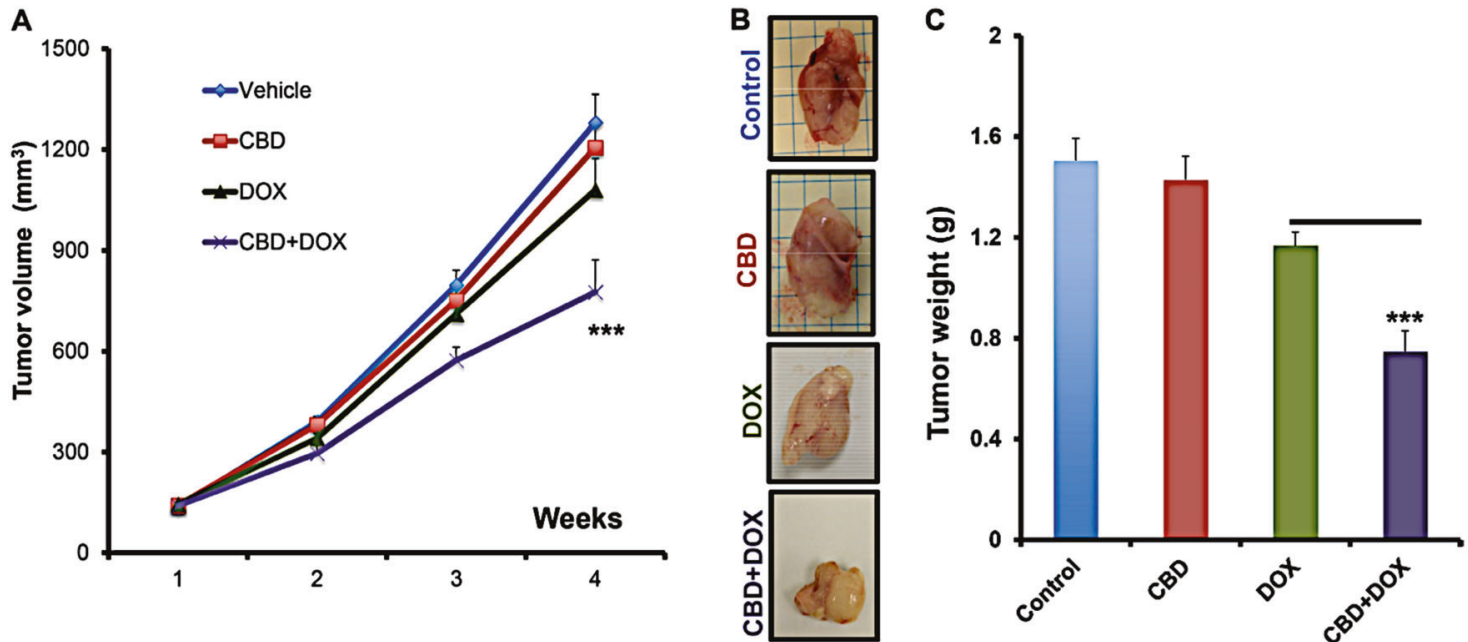

D

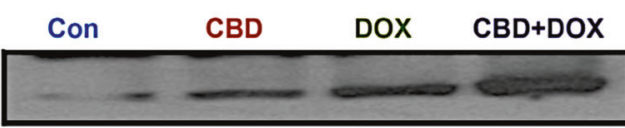

Cleaved PARP

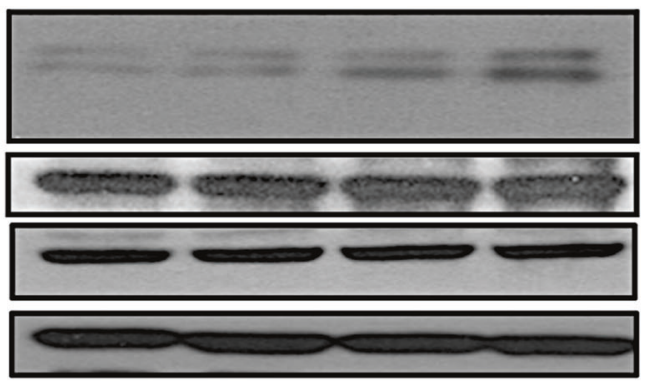

Cleaved caspase-3

$\beta$-Actin

TRPV2

GAPDH

Fig. 3 CBD improves the antitumor chemotherapeutic efficacy in vivo. Nude mice were orthotopically injected into 4th mammary gland with SUM159 cells and subjected to the indicated treatments for 4 weeks and the tumor volume a has been measured every week and the weight c of the dissected tumors has been determined for each group. b
Representative images of dissected tumors from the indicated experimental groups. d Tumor lysates from the experimental groups were used for western blot analysis and immunoblotted against the indicated proteins. Courtesy of Elbaz et al. [48] 
maintenance; its specific inhibitor, tranilast, could represent a potential targeted therapeutic agent against ESCCs [51].

\section{Role for TRPV2 in benign hepatoma and in hepatocarcinomas}

Oxidative stress is involved in cancer initiation and progression. TRPV2-mediated oxidative stress in human hepatoma (HepG2 and Huh-7) cell lines has been reported [52]. TRPV2 mRNA overexpression has been found in $\mathrm{H}_{2} \mathrm{O}_{2}$-exposed $\mathrm{HepG} 2$ and Huh-7 cells [52]. Increased TRPV2 levels in $\mathrm{H}_{2} \mathrm{O}_{2}$-treated hepatoma cells, potentiating the Akt and Nrf2 oxidative-stress inhibition, enhanced the p38/JNK1 MAPK activation and cell death, suggesting that TRPV2 may act by increasing the oxidative stress-induced cytotoxicity and drug resistance in cancers [52].

The relationship between TRPV2 expression in human hepatocellular carcinoma (HCC), its role in hepatocarcinogenesis and the TRPV2 clinical significance has been also studied. qRT-PCR and immunoblotting revealed an increased TRPV2 $\mathrm{mRNA}$ and protein in $\sim 30 \%$ of HCC cases and a statistical significant relation between TRPV2 expression, portal vein invasion and differentiation level [53]. The hepatocarcinoma phenotype is driven by liver cancer stem-like cells (LCSLCs) subpopulations CD133 ${ }^{+}$,
$\mathrm{CD} 90^{+}, \mathrm{CD} 44^{+}, \mathrm{CD} 13^{+}$, and $\mathrm{CD} 24^{+}$, endowed of selfrenewal [54]. Knockdown of TRPV2 in HepG2 cells increased the CD133, CD44, and ALDH1 expression and spheroid and colony formation, whereas the contrary effects were found in TRPV2-SMMC-7721 enforced cells. Furthermore, TRPV2 overexpression reverted the inhibition of spheroid and colony formation, whereas TRPV2 downregulation reduced the stem cell expression in HepG2 cells. Probenecid and tranilast suppressed and increased respectively spheroid and colony formation in LCSLCs and liver cancer cell lines [50] as well as inhibited or promoted tumor growth of HepG2 xenografts in the severe combined immunodeficient (SCID) mouse model, respectively. Similarly, downregulation of CD133, CD44, and ALDH1 in TRPV2 knockdown liver cancer cells was reported (Fig. 4) [44].

\section{TRPV2 channels in brain tumors: role of TRPV2 in gliomagenesis and glioma stem cells}

TRPV2 mRNA and protein expression was found in normal human astrocytes, in U87MG cells, in primary glioma cells (MZC, FCL, and FSL lines) and glioma tissues; in particular, TRPV2 levels declined as histological grade

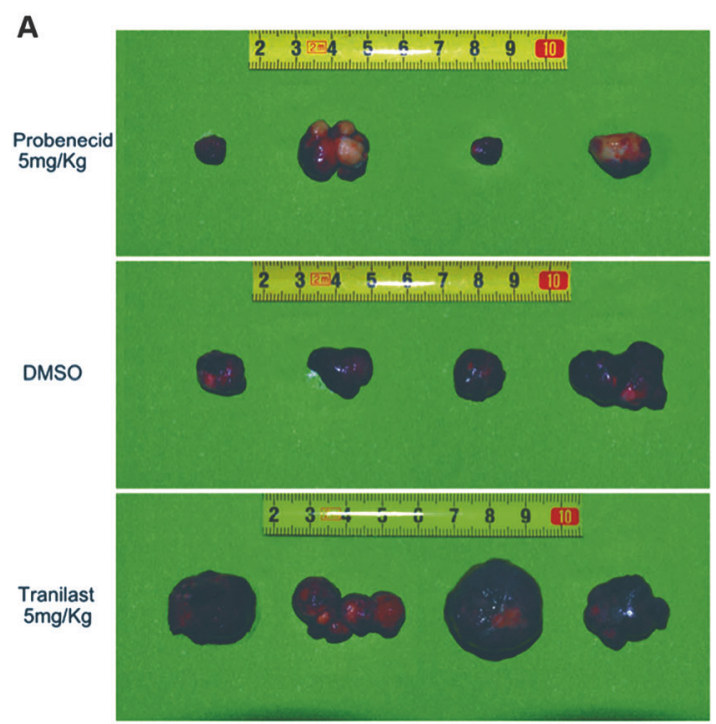

Fig. 4 In in vivo effects of probenecid and tranilast on the tumor growth of HepG2 SCID mouse xenografts. a Images of HepG2 xenografts following treatment of the mice with $5.0 \mathrm{mg} / \mathrm{kg}$ probenecid and tranilast. b Relative tumor volume of HepG2 xenografts in the SCID mouse model following treatment with $5.0 \mathrm{mg} / \mathrm{kg}$ probenecid and tranilast. Statistical analysis was conducted for the comparison of the SCID mice that were treated with $5.0 \mathrm{mg} / \mathrm{kg}$ tranilast, the animals treated with DMSO and those treated with $5.0 \mathrm{mg} / \mathrm{kg}$ of probenecid $* p<0.05, * * p<0.01$. c Tumor weight of HepG2 xenografts in the SCID mouse model following treatment with $5.0 \mathrm{mg} / \mathrm{kg}$ probenecid

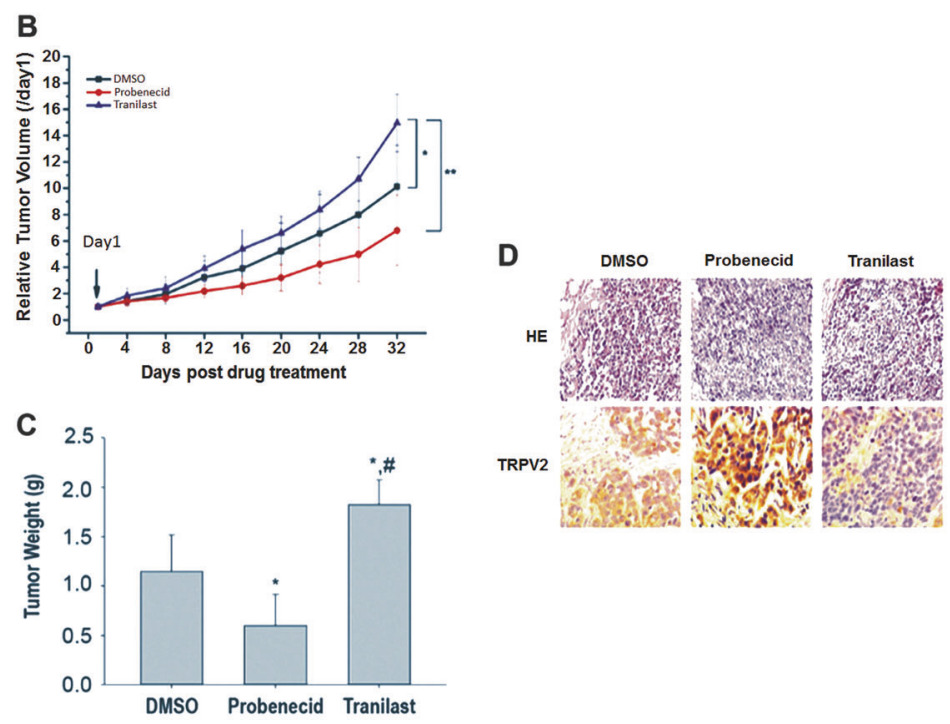

and tranilast. Statistical analysis was conducted for the comparison of HepG 2 cells treated with $0.1 \%$ DMSO, ${ }^{*} p<0.05$. HepG 2 cells treated with probenecid $(5.0 \mathrm{mg} / \mathrm{Kg})$ were compared with HepG2 cells treated with tranilast $(5.0 \mathrm{mg} / \mathrm{Kg}),{ }^{\#} p<0.05$. d The histological morphology and TRPV2 protein expression levels of HepG2 xenografts in the SCID mouse model following treatment of the animals with $5.0 \mathrm{mg} / \mathrm{kg}$ probenecid and tranilast. The histological evaluation was determined by $H \& E$ staining and immunohistochemistry. Courtesy of $\mathrm{Hu}$ et al. [44] 
increased ( $n=49$ cases) [55]. A marked downregulation of Fas and procaspase- 8 mRNA expression in association with upregulation of cyclin E1, cyclin-dependent kinase 2, E2F1 transcription factor-1, V-raf-1 murine leukemia viral oncogene homolog 1, and $\mathrm{Bcl}-2$-associated $\mathrm{X}$ protein mRNA expression, was evidenced in TRPV2 silenced (siTRPV2) U87MG cells. Furthermore, increased proliferation and rescued glioma cells to Fas-induced apoptosis, in an ERKdependent manner was evidenced in siTRPV2-U87MG cells. Indeed, treatment of siTRPV2-U87MG cells with PD98059, a specific MEK inhibitor, reduced Bcl-X(L), promoted CD95 expression and restoring Akt/PKB activation resulted in low U87MG cell proliferation, and increased CD95-mediated apoptosis. Enforced TRPV2 expression in primary glioma cells, by triggering CD95 overexpression, reduced cell viability and enhanced spontaneous and CD95-dependent apoptosis. Overall, in glioma cells, TRPV2 negatively controls the survival and proliferation, as well as resistance to CD95-induced apoptosis in ERK-dependent manner [55]. GBM are characterized by high invasiveness, proliferation, and drug resistance. Chemotherapy with temozolomide (TMZ), carmustine (BCNU) or DOXO was used in GBM treatment; however, their efficacy is limited. In this regard, TRPV2 activation leads to inhibition of GBM proliferation and overcoming of the BCNU resistance [56]. TRPV2 can be activated by CBD; this agonist increases drug uptake and cooperates with cytotoxic agents to induce $\mathrm{Ca}^{2+}$ flux and apoptosis of glioma cells, but not of normal astrocytes. In addition, deletion of TRPV2 pore domain, by affecting on channel permeation, inhibits $\mathrm{CBD}$-induced $\mathrm{Ca}^{2+}$ rise, drug uptake and cytotoxic effects [56]. Furthermore, a significant relationship between TRPV2 overexpression and survival of GBM patients was reported by Alptekin et al. that demonstrated for the first time that TRP channels, including TRPV2, contribute to the progression/survival of GBM patients [57]. CSCs are considered as the origin of cancer. They represent a small population within the tumor mass, dynamically interchanging between a quiescent and proliferative state in response to stress or DNA damage. Elevated intra-tumor heterogeneity, diffuse parenchymal metastasis and resistance to chemotherapies, result in cancer relapse often leading to patient death. In brain tumors, CSCs seem to derive from neural/progenitor stem cells, and a relevant role for TRPV2 in neurogenesis and gliomagenesis has been suggested [58, 59].

In GBM, recent evidence indicates that cannabinoids inhibit gliomagenesis and promote glioblastoma stem-like cells (GSCs) differentiation. Data reported by Morelli et al. revealed TRPV2 expression in human GSCs suggesting a role in aberrant GSC differentiation. Treatment of GSC lines with RR, EDTA, or knockdown of TRPV2 gene during differentiation, decreases GFAP and $\beta$ (III)-tubulin expression, conversely, phorbol ester, such as phorbol-12myristate-13-acetate, reduces TRPV2 expression, enhances GSCs proliferation, and reverts the differentiation. Forced TRPV2 expression in GSC lines enhanced GFAP and $\beta$ (III)-tubulin levels and inhibited the proliferation. In a xenograft mouse model, TRPV2 over-expression inhibits GSCs proliferation and promotes a glial phenotype differentiation. Therefore, deeper knowledge of TRPV2 molecular pathways in GSCs could improve the specificity and efficacy of the pharmacological approaches [43]. GSCs shown high radio- and chemoresistance are responsible of the recurrence and relapse of GBM. At present, druginduced differentiation is an interesting approach to redifferentiated CSC subpopulations. CBD by stimulating TRPV2 triggers the GSCs differentiation by inducing autophagy and inhibiting GSCs proliferation and clonogenicity. Moreover, the $\mathrm{CBD}$ and $\mathrm{BCNU}$ combination overcame the GSC resistance to BCNU treatment, by inducing apoptosis [10]. Recently a role for Acute myeloid leukemia (Aml-1/Runx-1) transcription factor in GBM proliferation/differentiation has been demonstrated. In GSCs, three Aml-1 spliced variants (Aml-1a, b, and c) have been discovered to be modulated in their expression during GSC differentiation. In this regard, upregulation of Aml-1a form was observed during GSCs differentiation; and Aml1a downregulation restores a stem cell immature phenotype in differentiated GSCs. The Aml-1a binds the TRPV2 promoters and CBD upregulated its expression in a TRPV2and PI3K/AKT-dependent manner [10]. These data are particularly relevant in the view of the effects showed by CBD on the endothelial cells from blood-brain barrier (BBB). In these cells, CBD induces proliferation, migration, and tubulogenesis suggesting that it might be a potent target for modulating the $\mathrm{BBB}$ functions. Moreover, CBDinduced intracellular $\mathrm{Ca}^{2+}$ rise, increased cerebral endothelial human CMEC/D3 cell numbers, and increased the transendothelial electrical resistance of brain microvessel endothelial cells [60].

Accordingly, Perálvarez-Marín's group, using a systematic proteomics and computational analysis approach, confirmed the clinical relevance of TRPV2 overexpression in GBM, with the discovery of the TRPV2 interactome (Fig. 5) [61], thus opening new perspectives to identify new biomarkers for diagnosis, prognosis, and therapeutics in GBM. The TRPV2 interactome consists of TRPV2 plus 22 proteins that includes: transport proteins (TRPV2, KCNJ10, CACNG3, and SLC44A2), catalytic proteins (ALDH1A3, INPP5F, and PIP4K2B), trafficking proteins (ARF1, ARL15, ABR, SYT-9, and SNAPIN), signaling molecules (FGF1, ST18, SHISA6, CDH6, and SDC3), myelin components (PLP1, Opalin, and NTM), lipid metabolism proteins (SACM1L, PEBP1, INPP5F, and PIP4K2B), etc. In the TRPV2 interactome, the strongest protein interaction with 
A
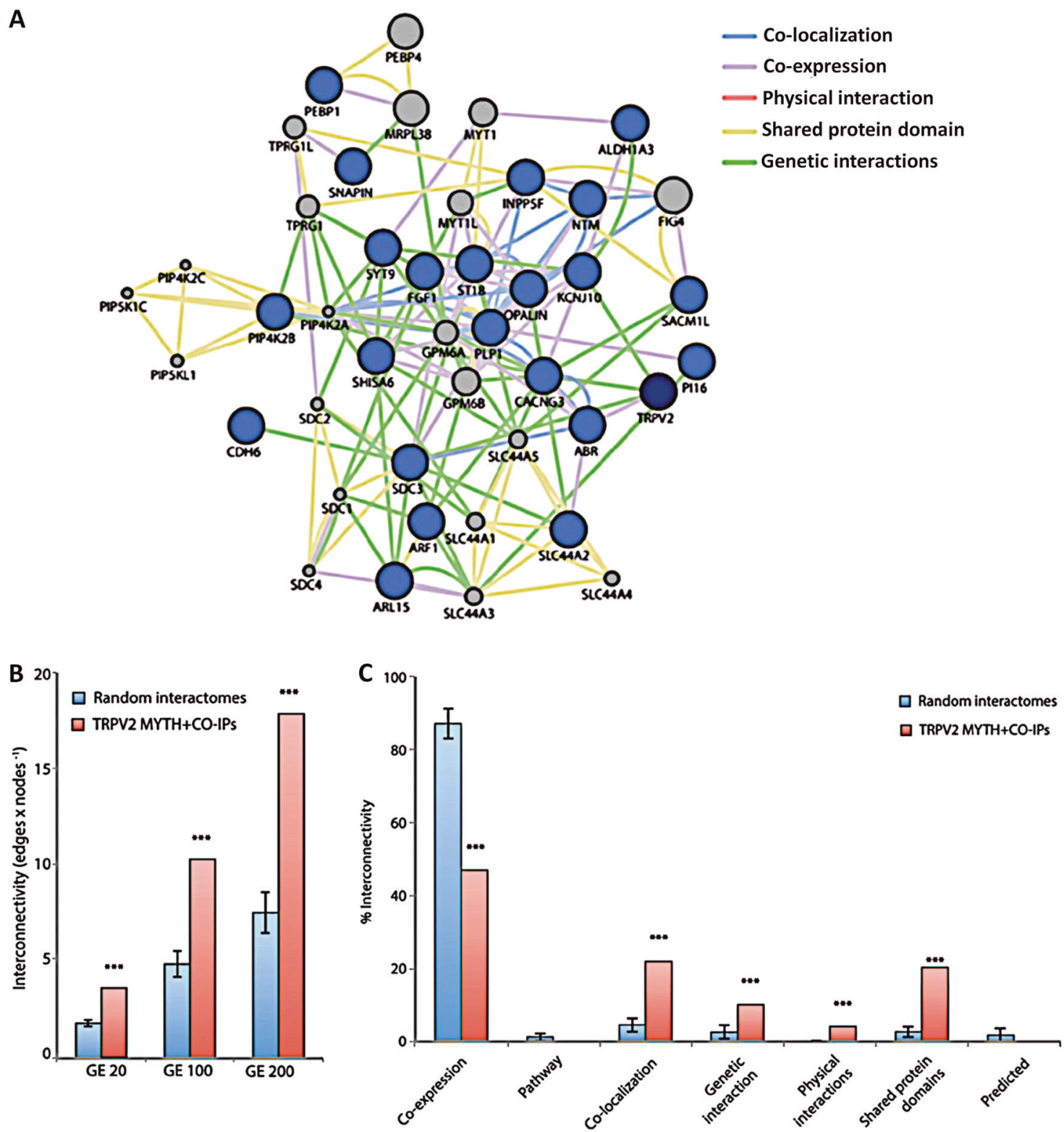

Fig. 5 TRPV2 interactome in GBM. a Gene enrichment using the 20 closest related genes. The network generated connects TRPV2 plus CoIP interactors (blue dots) and 20 closest related genes (grey dots) according with the following GO-terms: gene co-expression (purple lines); co-localization (blue lines); genetic interactions (green lines); physical interactions (red lines) and shared domains (brown lines). b Network interconnectivity measured as the ratio of edges: nodes within the network. TRPV2 interactome from MYTH plus CoIP was

TRPV2 was reported for ABR, ARL15, NTM, Opalin, SACM1L, and ST18 proteins [61]. Neoplasms and diseases of the nervous system are strictly associated in the TRPV2 interactome, with the expression of ABR, FGF1, KNJ10, PEBP1, PLP1, and SOC3 proteins [43]. The TRPV2 interactome-based signature allows to discriminate among high- and low-risk GBM, in terms of overall survival (OS), with less survival in GBM patients expressing the TRPV2 protein interactome. Indeed, GBM patients with high TRPV2 compared with randomly generated networks $(n=16)$. The analysis was performed using a gene enrichment of 20, 100, or 200 closest related genes (GE 20, GE100, and GE200, respectively). c. The nature of the network interconnectivity was assessed for TRPV2 interactome and randomly generated networks. The percentage of each type of connection over total connections within the network was represented. $* * * p$ values $<0.001$ between compared groups. Courtesy of DonateMacian et al. [61]

interactome protein expression, show a negative prognosis, tumor progression, recurrence, and TMZ-resistance [61].

\section{Expression and function of TRPV 2 in the hematologic malignancies}

The TRP channels mediate $\mathrm{Ca}^{2+}$ influx also in nonexcitable cells, such as in normal and neoplastic cells from 
hematopoietic tissues. Several data evidenced that TRP expression was altered in hematologic malignancies: multiple myeloma (MM), acute, and chronic myeloid and lymphoid leukemia (AML, ALL, CML and CLL) and Hodgkin and non-Hodgkin, B- and T-lymphomas [62]. These effects are the result of the widespread roles played by the different subfamily of the TRP channels on hematopoietic cell proliferation, differentiation and apoptosis. The analysis of TRPV2 expression in different hematological malignancies, showed the expression of TRPV2 both in the myeloid and lymphoid leukemia [62]; moreover a very peculiar high TRPV2 expression in B cell lymphomas and MM [63, 64], that parallel the restricted TRPV2 expression in normal immune cells compared with its expression in other human body tissues was reported [65].

$\mathrm{MM}$ is a plasma cell (PC) malignancy characterized by the accumulation in the bone marrow of a monoclonal PC type. TRPV2 activation, induced by treatment of MM cells with CBD, decreases the proliferation and stimulates drug-induced cell death in human MM cells.

MM heterogeneity was evidenced by Morelli et al., with the $\mathrm{CD} 138^{+} \mathrm{TRPV}^{+}$and $\mathrm{CD} 138^{+} \mathrm{TRPV} 2-\mathrm{PC}$ subpopulations expressed in MM patients, and the CD138 ${ }^{+}$TRPV2population only present in RPMI8226 and U266 MM cell lines analyzed [66]. In these cells, CBD alone or in synergy with bortezomib, normally used in MM treatment, strongly arrested cell cycle progression and induced cell death in CD138 ${ }^{+}$TRPV2- MM cells and MM cells transfected with TRPV2 (CD138 ${ }^{+} \mathrm{TRPV}^{+}$), by regulating the ERK, AKT, and NF-KB pathways, with more relevant effects in TRPV2positive cells. Collectively, these findings suggest the use of $\mathrm{CBD}$ as adjuvant to increase the proteasome inhibitor activity in MM [66].

Myeloma causes bone destruction and increase $\mathrm{Ca}^{2+}$ levels in marrow microenvironment by triggering osteoclastic differentiation (Myeloma bone disease; MBD). Osteolysis during MBD, requires high TRPV2 expression and calcium flux. TRPV2 modulates the nuclear factor- $\mathrm{kB}$ ligand (RANKL)-dependent osteoclastic differentiation by activating the $\mathrm{Ca}^{2+}$-calcineurin-nuclear factor of activated $\mathrm{T}$ cells signaling pathway, and systemic administration of SKF96365, a TRPV2 inhibitor, reduced the MM-induced osteoclast formation and osteolysis [67].

Pottosin et al. have recently reported the TRPV2 expression in human leukemic cells. TRPV2 is expressed in Jurkat $\mathrm{T}$ lymphoblasts, and its effects were blocked by amiloride, $\mathrm{Gd}^{3+}$, RR, and SKF96365. TRPV2 silencing abolished the stretch-activated current in Jurkat $\mathrm{T}$ lymphoblasts, suggesting a role for TRPV2 in lymphocyte $\mathrm{Ca}^{2+}$ signaling in AML disease [68].

Leukemia patients, treated with chemotherapeutic drugs, showed an increased risk of pulmonary complications as the result of LBCs infiltration into the lung parenchyma. In this regard, LBCs showed increased TRPV2 expression compared with normal human peripheral blood mononuclear cells (PBMCs), which is correlated with increase oncogenic activity. In addition, upregulation of full-length isoform and downregulation of the nonfunctional short form pore-less variant of TRPV2 protein were found in LBCs, whereas the opposite effects were evidenced in PBMCs. It was also demonstrated that silencing or pharmacological targeting of TRPV2 by tranilast or SKF96365, enhances cell cycle arrest and caspase-dependent apoptosis [39]. Tranilast and SKF96365 inhibit the expression of chemotactic peptide that induces TRPV2-dependent migration and invasion and downregulates the CD38 surface expression, responsible for leukemia and lung airway inflammation. Overall, TRPV2 could represent a potential biomarker for patients with leukemia (with lung inflammation) and tranilast could be used as an adjuvant therapeutic strategy in these patients [39].

\section{Expression of TRPV2 in other cancer types}

The expression of TRPV2 in gastric cancer (GC) patients has been recently analyzed [58]. Among GC patients investigated, high levels of TRPV2 expression was associated with short OS. The expression of TRPV2 increases as tumor stage increase. In addition, GC showed a higher TRPV2 transcripts and protein levels compared with normal stomach specimens. Moreover, in Lauren's intestinal GCs and patients treated with adjuvant therapy, the TRPV2 levels positively associate with poor prognosis [69].

TRPV2 expression was also evaluated in epidermal melanocytes, two human malignant melanoma (A375, G361) and two metastatic melanoma (A2058, SK-MEL-3) cell lines [70]. Results evidenced that TRPV2, together with TRPV4, TRPA1, and TRPM8 was ectopically expressed in melanocytes and melanoma cells. TRPV2 channel activation reduced cell viability in melanoma A2058 and A375 cells. The 2-APB TRPV2 agonist induced cell necrosis in A2058 cells, suggesting that TRPV2 expression may serve as biomarker for targeting therapeutic drugs and for prognosis in melanomas [70]. Abnormal expression of TRPV channels has been also reported in meningiomas, tumors likely originating from arachnoid cap cells. A significant correlation between the TRPV1, TRPV2, TRPV3, and TRPV4 expression in different meningioma subtypes was evidenced [71].

\section{Cancer adverse effects: role for the TRPV2 channel}

Muscle wasting during cancer cachexia causes loss of body and skeletal muscle weight and diminished muscle force and 
locomotor activity, so contributing to patient morbidity [72]. Cachexia of muscles results in myolysis, reduced sialic acid content of sarcolemma, enhanced lysosomal exocytosis, and sarcolemma localization of phosphorylated $\mathrm{Ca}^{2+} /$ calmodulindependent protein kinase II, abnormal autophagy with increased levels of autophagic markers and degradation of dystrophin [61]. Because of the role of TRPV2 in muscle dystrophy, a potential involvement of TRPV2 in cachexiainduced muscle damage has been considered. Studies in a murine model of cancer cachexia in mice bearing Lewis lung carcinoma/colon adenocarcinoma evidenced a slight accumulation of TRPV2 and TRPV2-dependent $\mathrm{Ca}^{2+}$ influx was low in cachexia-associated myotubes and cachexia was not ameliorated by dominant-negative inhibition of TRPV2 [73], suggesting a TRPV2-independent mechanisms mediating muscle damage in cancer cachexia.
Dysfunction of TRP channels contributes to the thermal hypersensitivity that accompanies painful conditions in several inflammatory and cancer diseases. Classical thermosensitive TRP channels are TRPV1, TRPV2, TRPV3, TRPV4, TRPM3, TRPM8, and TRPA1 [74]. In addition, althoughTRPV2 is also required for mechanical nociception and the stretch-evoked response of primary sensory neurons [75], no data on the analgesic effects of this compound or its derivates have been provided in cancer [76]. A role for TRPV2 in outgrowth of sensory neurons under neurothrophin-regulated signaling has been suggested [5]. However, although neurothrophin-3 (NT-3) has been found to alleviate perineural invasion and abdominal pain in pancreatic ductal adenocarcinoma patients (PDACs) and high levels of TRPV2 as well as the TRPV1 pain-related proteins were identified, no correlation between TRPV2 and
Table 1 Expression and functions of TRPV2 channels in different human cancer types

\begin{tabular}{|c|c|c|c|}
\hline Cancer type & TRPV2 & Effect & References \\
\hline \multirow[t]{3}{*}{$\mathrm{UC}$} & Increase & $(+)$ malignancy & [29] \\
\hline & Activation & $(-)$ viability, apotosis & {$[30]$} \\
\hline & Overexpression & $(+)$ migration, invasion & {$[31]$} \\
\hline $\mathrm{PCa}$ & Activation & $(+)$ migration, invasion & {$[33,34,36,37]$} \\
\hline \multirow[t]{4}{*}{$\mathrm{BC}$} & Inhibition & $(-)$ proliferation & [40] \\
\hline & Activation & $(-)$ proliferation & [42] \\
\hline & Inhibition & $(-)$ migration & {$[47]$} \\
\hline & Increase & $(+)$ chemiosensitivity apoptosis & {$[48]$} \\
\hline \multirow[t]{2}{*}{ ESC } & Increase & $(+)$ progression & {$[49,50]$} \\
\hline & Increase & $(+)$ stemness & {$[51]$} \\
\hline \multirow[t]{2}{*}{$\mathrm{HCC}$} & Increase & $(+)$ portal vein invasion & {$[53]$} \\
\hline & Inhibition & $(+)$ stemness, tumor growth & {$[44]$} \\
\hline \multirow[t]{6}{*}{ GBM } & Inhibition & (-) Fas apoptosis & {$[55]$} \\
\hline & Activation & $(+)$ drug uptake, apoptosis & [56] \\
\hline & Increase & progression & [57] \\
\hline & Activation & $\begin{array}{l}(+) \text { GSC differentiation Runx-1 activation } \\
(+) \text { drug sensitivity }\end{array}$ & {$[10,43]$} \\
\hline & Increase & $(-)$ proliferation & [43] \\
\hline & & $(+)$ angiogenesis & {$[47]$} \\
\hline MM & Activation & $(-)$ proliferation $(+)$ cell death & {$[66]$} \\
\hline \multirow[t]{3}{*}{ ALL } & Inhibition & $(-)$ stretch-activated current & {$[68]$} \\
\hline & Inhibition & $(+)$ cycle arrest, apoptosis & [39] \\
\hline & & $(-)$ migration, invasion & \\
\hline GC & Increase & $(+)$ progression & [69] \\
\hline \multirow[t]{2}{*}{$\mathrm{ME}$} & Activation & (-) viability & {$[70]$} \\
\hline & & $(+)$ necrosis & \\
\hline
\end{tabular}

$(+)$ promote; $(-)$ inhibit

$U C$ Urothelial cancer, $P C a$ prostate cancer, $B C$ breast cancer, ESCC esophageal squamous cancer, $H C C$ hepatocarcinoma, $G B M$ glioblastoma maligno, $M M$ multiple myeloma, $A L L$ acute lymphoblastic leukemia, $G C$ gastric cancer, $M E$ melanoma, CSC cancer stem cell

TRPV2 channels control multiple processes involved in cancer progression by modulating survival, cell proliferation, angiogenesis, migration, and invasion in different cancer types. Clinical evidence demonstrates in glioblastoma, epatocarcinoma, and urogenital cancer a strictly relation between altered TRPV2 expression and negative prognosis. Thus, TRPV2 represents a novel prognostic biomarker for cancers 
NT-3 pathway was identified in PDAC patients [77]. Moreover, Yamamoto et al., in a mouse model of bone cancer, have demonstrated that TRPV2 is involved in movement-related pain but not in ongoing pain [78].

\section{Conclusions}

TRPV2 exhibits an oncogenic activity in different types of cancer associated with changes in its expression levels (i.e., mainly TRPV2 overexpression) (Table 1). Since, it has been demonstrated by several reports that TRPV2 affects signaling pathways responsible for cancer growth/proliferation, migration/invasion, and apoptosis, TRPV2 represents a novel promising pharmacologic target especially in the management of the most aggressive cancers.

A TRPV2 interactome signature has been identified whose overexpression is associated with reduced OS, drug resistance and poor prognosis in GBM patients. Clinical studies are now required to confirm TRPV2 as a useful and sure molecular biomarker to be employed in the patient selection in order to promote a targeted therapy. Interestingly, tranilast, a TRPV2 inhibitor, was found to be effective in leukemia cells and primary blast so much so that it is now considered a potential candidate for further preclinical studies alone or in combination regimens. On the contrary, in UCs no experimental, preclinical, or clinical studies using tranilast have been provided so far. The clinical usage of them in murine cancer model as well as in preclinical studies could be essential to highlight the importance for targeting TRPV2 in high-risk cancer patients to propose a new therapeutic approach.

Acknowledgements This work was supported by Fondazione Umberto Veronesi (Postdoctoral Fellowship 2019) to MBM.

\section{Compliance with ethical standards}

Conflict of interest The authors declare that they have no conflict of interest.

Publisher's note Springer Nature remains neutral with regard to jurisdictional claims in published maps and institutional affiliations.

\section{References}

1. Venkatachalam K, Montell C. TRP Channels. Annu Rev Biochem. 2007;76:387-417.

2. Caterina MJ, Schumacher MA, Tominaga M, Rosen TA, Levine JD, Julius D. The capsaicin receptor: a heat-activated ion channel in the pain pathway. Nature. 1997;389:816-24.

3. Caterina MJ, Rosen TA, Tominaga M, Brake AJ, Julius D. A capsaicin-receptor homologue with a high threshold for noxious heat. Nature. 1999;398:436-41.
4. Perálvarez-Marín A, Doñate-Macian P, Gaudet R. What do we know about the transient receptor potential vanilloid 2 (TRPV2) ion channel? FEBS J. 2013;280:5471-87.

5. Cohen MR, Johnson WM, Pilat JM, Kiselar J, DeFrancescoLisowitz A, Zigmond RE, et al. Nerve growth factor regulates transient receptor potential vanilloid 2 via extracellular signalregulated kinase signaling to enhance neurite outgrowth in developing neurons. Mol Cell Biol. 2015;35:4238-52.

6. Cohen MR, Huynh KW, Cawley D, Moiseenkova-Bell VY. Understanding the cellular function of TRPV2 channel through generation of specific monoclonal antibodies. PLoS ONE. 2013;8: e85392.

7. Park U, Vastani N, Guan Y, Raja SN, Koltzenburg M, Caterina MJ. TRP vanilloid 2 knock-out mice are susceptible to perinatal lethality but display normal thermal and mechanical nociception. $\mathrm{J}$ Neurosci. 2011;31:11425-36.

8. Bang S, Kim KY, Yoo S, Lee S-H, Hwang SW. Transient receptor potential V2 expressed in sensory neurons is activated by probenecid. Neurosci Lett. 2007;425:120-5.

9. Huynh KW, Cohen MR, Chakrapani S, Holdaway HA, Stewart PL, Moiseenkova-Bell VY. Structural insight into the assembly of TRPV channels. Structure. 2014;22:260-8.

10. Nabissi M, Morelli MB, Amantini C, Liberati S, Santoni M, RicciVitiani L, et al. Cannabidiol stimulates Aml-1a-dependent glial differentiation and inhibits glioma stem-like cells proliferation by inducing autophagy in a TRPV2-dependent manner. Int J Cancer. 2015;137:1855-69.

11. Mercado J, Gordon-Shaag A, Zagotta WN, Gordon SE. Ca2+dependent desensitization of TRPV2 channels is mediated by hydrolysis of phosphatidylinositol 4,5-bisphosphate. J Neurosci. 2010;30:13338-47.

12. Huynh KW, Cohen MR, Jiang J, Samanta A, Lodowski DT, Zhou $\mathrm{ZH}$, et al. Structure of the full-length TRPV2 channel by cryo-EM. Nat Commun. 2016;7:11130.

13. Zubcevic L, Le S, Yang H, Lee S-Y. Conformational plasticity in the selectivity filter of the TRPV2 ion channel. Nat Struct Mol Biol. 2018;25:405-15.

14. Dosey TL, Wang Z, Fan G, Zhang Z, Serysheva II, Chiu W, et al. Structures of TRPV2 in distinct conformations provide insight into role of the pore turret. Nat Struct Mol Biol.2019;26:40-9.

15. Shibasaki K. Physiological significance of TRPV2 as a mechanosensor, thermosensor and lipid sensor. J Physiol Sci. 2016;66: 359-65.

16. Li Y, Li Q, Zhang O, Guan X, Xue Y, Li S, et al. miR-202-5p protects rat against myocardial ischemia reperfusion injury by downregulating the expression of Trpv2 to attenuate the Ca $2+$ overload in cardiomyocytes. J Cell Biochem. 2019;120:13680-93.

17. Iwata Y, Matsumura T. Blockade of TRPV2 is a novel therapy for cardiomyopathy in muscular dystrophy. Int J Mol Sci. 2019; 20:3844.

18. Iwata Y, Katayama Y, Okuno Y, Wakabayashi S. Novel inhibitor candidates of TRPV2 prevent damage of dystrophic myocytes and ameliorate against dilated cardiomyopathy in a hamster model. Oncotarget. 2018;9:14042-57.

19. Matsumura T, Matsui M, Iwata Y, Asakura M, Saito T, Fujimura $\mathrm{H}$, et al. A pilot study of tranilast for cardiomyopathy of muscular dystrophy. Intern Med. 2018;57:311-8.

20. Sawatani T, Kaneko YK, Doutsu I, Ogawa A, Ishikawa T. TRPV2 channels mediate insulin secretion induced by cell swelling in mouse pancreatic beta-cells. Am J Physiol Cell Physiol. 2019;316:C434-43.

21. Sun W, Li C, Zhang Y, Jiang C, Zhai M, Zhou Q, et al. Gene expression changes of thermo-sensitive transient receptor potential channels in obese mice. Cell Biol Int. 2017;41:908-13.

22. D'Agnelli S, Arendt-Nielsen L, Gerra MC, Zatorri K, Boggiani L, Baciarello $\mathrm{M}$, et al. Fibromyalgia: genetics and epigenetics 
insights may provide the basis for the development of diagnostic biomarkers. Mol Pain. 2019;15:174480691881994.

23. Park D-J, Kim S-H, Nah S-S, Lee JH, Kim S-K, Lee Y-A, et al. Polymorphisms of the TRPV2 and TRPV3 genes associated with fibromyalgia in a Korean population. Rheumatology. 2016;55:1518-27.

24. Leveque M, Penna A, Le Trionnaire S, Belleguic C, Desrues B, Brinchault G, et al. Phagocytosis depends on TRPV2-mediated calcium influx and requires TRPV2 in lipids rafts: alteration in macrophages from patients with cystic fibrosis. Sci Rep. 2018;8:4310.

25. Link TM, Park U, Vonakis BM, Raben DM, Soloski MJ, Caterina MJ. TRPV2 has a pivotal role in macrophage particle binding and phagocytosis. Nat Immunol. 2010;11:232-9.

26. Liberati S, Morelli MB, Amantini C, Santoni M, Nabissi M, Cardinali $\mathrm{C}$, et al. Advances in transient receptor potential vanilloid-2 channel expression and function in tumor growth and progression. Curr Protein Pept Sci. 2014;15:732-7.

27. Liberati S, Morelli M, Amantini C, Farfariello V, Santoni M, Conti A, et al. Loss of TRPV2 Homeostatic Control of Cell Proliferation Drives Tumor Progression. Cells. 2014;3:112-28.

28. Gkika D, Prevarskaya N. Molecular mechanisms of TRP regulation in tumor growth and metastasis. Biochim Biophys Acta. 2009;1793:953-8.

29. Caprodossi S, Lucciarini R, Amantini C, Nabissi M, Canesin G, Ballarini $\mathrm{P}$, et al. Transient receptor potential vanilloid type 2 (TRPV2) expression in normal urothelium and in urothelial carcinoma of human bladder: correlation with the pathologic stage. Eur Urol. 2008;54:612-20.

30. Yamada T, Ueda T, Shibata Y, Ikegami Y, Saito M, Ishida Y, et al. TRPV2 activation induces apoptotic cell death in human T24 bladder cancer cells: a potential therapeutic target for bladder cancer. Urology. 2010;76:509.e1-509.e7.

31. Liu Q, Wang X. Effect of TRPV2 cation channels on the proliferation, migration and invasion of 5637 bladder cancer cells. Exp Ther Med. 2013;6:1277-82.

32. Mizuno H, Suzuki Y, Watanabe M, Sokabe T, Yamamoto T, Hattori R, et al. Potential role of transient receptor potential (TRP) channels in bladder cancer cells. J Physiol Sci. 2014;64:305-14.

33. Monet M, Gkika D, Lehen'kyi V, Pourtier A, Vanden Abeele F, Bidaux G, et al. Lysophospholipids stimulate prostate cancer cell migration via TRPV2 channel activation. Biochim Biophys Acta. 2009;1793:528-39.

34. Monet M, Lehen'kyi V, Gackiere F, Firlej V, Vandenberghe M, Roudbaraki M, et al. Role of cationic channel TRPV2 in promoting prostate cancer migration and progression to androgen resistance. Cancer Res. 2010;70:1225-35.

35. Sugio S, Nagasawa M, Kojima I, Ishizaki Y, Shibasaki K. Transient receptor potential vanilloid 2 activation by focal mechanical stimulation requires interaction with the actin cytoskeleton and enhances growth cone motility. FASEB J. 2017;31:1368-81.

36. Oulidi A, Bokhobza A, Gkika D, Vanden Abeele F, Lehen'kyi V, Ouafik L, et al. TRPV2 mediates adrenomedullin stimulation of prostate and urothelial cancer cell adhesion, migration and invasion. PLoS ONE. 2013;8:e64885.

37. Warrington JI, Richards GO, Wang N. The role of the calcitonin peptide family in prostate cancer and bone metastasis. Curr Mol Biol Reports. 2017;3:197-203.

38. Bernardini M, Brossa A, Chinigo G, Grolez GP, Trimaglio G, Allart L, et al. Transient receptor potential channel expression signatures in tumor-derived endothelial cells: functional roles in prostate cancer angiogenesis. Cancers. 2019;11:956.

39. Siveen KS, Prabhu KS, Parray AS, Merhi M, Arredouani A, Chikri M, et al. Evaluation of cationic channel TRPV2 as a novel biomarker and therapeutic target in Leukemia-Implications concerning the resolution of pulmonary inflammation. Sci Rep. 2019;9:1554.

40. Nie L, Oishi Y, Doi I, Shibata H, Kojima I. Inhibition of proliferation of MCF-7 breast cancer cells by a blocker of $\mathrm{Ca}(2+)$ permeable channel. Cell Calcium. 1997;22:75-82.

41. Kojima I, Nagasawa M. TRPV2: a calcium-permeable cation channel regulated by insulin-like growth factors. In: Liedtke WB, Heller S, editors.TRP ion channel function in sensory transduction and cellular signaling cascades. Chapter 7. Boca Raton, FL: CRC Press/Taylor \& Francis; 2007.

42. McAllister SD, Murase R, Christian RT, Lau D, Zielinski AJ, Allison J, et al. Pathways mediating the effects of cannabidiol on the reduction of breast cancer cell proliferation, invasion, and metastasis. Breast Cancer Res Treat. 2011;129:37-47.

43. Morelli MB, Nabissi M, Amantini C, Farfariello V, Ricci-Vitiani L, di Martino S, et al. The transient receptor potential vanilloid-2 cation channel impairs glioblastoma stem-like cell proliferation and promotes differentiation. Int J Cancer. 2012;131:E1067-77.

44. Hu Z, Cao X, Fang Y, Liu G, Xie C, Qian K, et al. Transient receptor potential vanilloid-type 2 targeting on stemness in liver cancer. Biomed Pharmacother. 2018;105:697-706.

45. Laragione T, Harris C, Gulko PS. TRPV2 suppresses Rac1 and RhoA activation and invasion in rheumatoid arthritis fibroblastlike synoviocytes. Int Immunopharmacol. 2019;70:268-73.

46. Gogebakan B, Bayraktar R, Suner A, Balakan O, Ulasli M, Izmirli M, et al. Do Fasudil and Y-27632 affect the level of transient receptor potential (TRP) gene expressions in breast cancer cell lines? Tumor Biol. 2014;35:8033-41.

47. Gambade A, Zreika S, Guéguinou M, Chourpa I, Fromont G, Bouchet AM, et al. Activation of TRPV2 and BKCa channels by the LL-37 enantiomers stimulates calcium entry and migration of cancer cells. Oncotarget. 2016;7:23785-800.

48. Elbaz M, Ahirwar D, Xiaoli Z, Zhou X, Lustberg M, Nasser MW, et al. TRPV2 is a novel biomarker and therapeutic target in triple negative breast cancer. Oncotarget. 2018;9:33459-70.

49. Zhou K, Zhang S-S, Yan Y, Zhao S. Overexpression of transient receptor potential vanilloid 2 is associated with poor prognosis in patients with esophageal squamous cell carcinoma. Med Oncol. 2014;31:17.

50. Huang R, Wang F, Yang Y, Ma W, Lin Z, Cheng N, et al. Recurrent activations of transient receptor potential vanilloid-1 and vanilloid-4 promote cellular proliferation and migration in esophageal squamous cell carcinoma cells. FEBS Open Bio. 2019;9:206-25.

51. Shiozaki A, Kudou M, Ichikawa D, Fujiwara H, Shimizu H, Ishimoto T, et al. Esophageal cancer stem cells are suppressed by tranilast, a TRPV2 channel inhibitor. J Gastroenterol. 2018;53:197-207.

52. Ma W, Li C, Yin S, Liu J, Gao C, Lin Z, et al. Novel role of TRPV2 in promoting the cytotoxicity of H2O2-mediated oxidative stress in human hepatoma cells. Free Radic Biol Med. 2015;89:1003-13.

53. Liu G, Xie C, Sun F, Xu X, Yang Y, Zhang T, et al. Clinical significance of transient receptor potential vanilloid 2 expression in human hepatocellular carcinoma. Cancer Genet Cytogenet. 2010;197:54-9.

54. Liu L-L, Fu D, Ma Y, Shen X-Z. The power and the promise of liver cancer stem cell markers. Stem Cells Dev. 2011;20:2023-30.

55. Nabissi M, Morelli MB, Amantini C, Farfariello V, Ricci-Vitiani L, Caprodossi S, et al. TRPV2 channel negatively controls glioma cell proliferation and resistance to Fas-induced apoptosis in ERKdependent manner. Carcinogenesis. 2010;31:794-803.

56. Nabissi M, Morelli MB, Santoni M, Santoni G. Triggering of the TRPV2 channel by cannabidiol sensitizes glioblastoma cells to cytotoxic chemotherapeutic agents. Carcinogenesis. 2013;34:48-57. 
57. Alptekin M, Eroglu S, Tutar E, Sencan S, Geyik MA, Ulasli M, et al. Gene expressions of TRP channels in glioblastoma multiforme and relation with survival. Tumour Biol. 2015;36:9209-13.

58. Gimple RC, Bhargava S, Dixit D, Rich JN. Glioblastoma stem cells: lessons from the tumor hierarchy in a lethal cancer. Genes Dev. 2019;33:591-609.

59. Santoni G, Amantini C. The transient receptor potential vanilloid type-2(TRPV2) ion channels in neurogenesis and gliomagenesis: cross-talk between transcriptionfactors and signaling molecules. Cancers. 2019;11: p11 E322.

60. Luo H, Rossi E, Saubamea B, Chasseigneaux S, Cochois V, Choublier N, et al. Cannabidiol increases proliferation, migration, tubulogenesis, and integrity of human brain endothelial cells through TRPV2 activation. Mol Pharm. 2019;16:1312-26.

61. Donate-Macian P, Gomez A, Degano IR, Peralvarez-Marin A. A TRPV2 interactome-based signature for prognosis in glioblastoma patients. Oncotarget. 2018;9:18400-9.

62. Morelli MB, Liberati S, Amantini C, Nabiss M, Santoni M, Farfariello $\mathrm{V}$, et al. Expression and function of the transient receptor potential ion channel family in the hematologic malignancies. Curr Mol Pharmacol. 2013;6:137-48.

63. Boyd RS, Jukes-Jones R, Walewska R, Brown D, Dyer MJS, Cain K. Protein profiling of plasma membranes defines aberrant signaling pathways in mantle cell lymphoma. Mol Cell Proteomics. 2009;8:1501-15.

64. Fabris S, Todoerti K, Mosca L, Agnelli L, Intini D, Lionetti M, et al. Molecular and transcriptional characterization of the novel 17p11.2-p12 amplicon in multiple myeloma. Genes Chromosomes Cancer. 2007;46:1109-18.

65. Santoni G, Farfariello V, Liberati S, Morelli MB, Nabissi M, Santoni M, et al. The role of transient receptor potential vanilloid type- 2 ion channels in innate and adaptive immune responses. Front Immunol. 2013;4:34.

66. Morelli MB, Offidani M, Alesiani F, Discepoli G, Liberati S, Olivieri A, et al. The effects of cannabidiol and its synergism with bortezomib in multiple myeloma cell lines. A role for transient receptor potential vanilloid type-2. Int J Cancer. 2014;134:2534-46.

67. Bai H, Zhu H, Yan Q, Shen X, Lu X, Wang J, et al. TRPV2induced $\mathrm{Ca}(2+)$-calcineurin-NFAT signaling regulates differentiation of osteoclast in multiple myeloma. Cell Commun Signal. 2018;16:68.
68. Pottosin I, Delgado-Enciso I, Bonales-Alatorre E, Nieto-Pescador MG, Moreno-Galindo EG, Dobrovinskaya O. Mechanosensitive $\mathrm{Ca}(2)(+)$-permeable channels in human leukemic cells: pharmacological and molecular evidence for TRPV2. Biochim Biophys Acta. 2015;1848(1 Pt A):51-9.

69. Zoppoli P, Calice G, Laurino S, Ruggieri V, La Rocca F, La Torre G, et al. TRPV2 calcium channel gene expression and outcomes in gastric cancer patients: a clinically relevant association. J Clin Med. 2019;8:pii E622.

70. Zheng J, Liu F, Du S, Li M, Wu T, Tan X, et al. Mechanism for regulation of melanoma cell death via activation of thermoTRPV4 and TRPV2. J Oncol. 2019;2019:7362875.

71. Goutsou S, Tsakona C, Polia A, Moutafidi A, Zolota V, Gatzounis $\mathrm{G}$, et al. Transient receptor potential vanilloid (TRPV) channel expression in meningiomas: prognostic and predictive significance. Virchows Arch. 2019;475:105-14.

72. Fearon K, Strasser F, Anker SD, Bosaeus I, Bruera E, Fainsinger RL, et al. Definition and classification of cancer cachexia: an international consensus. Lancet Oncol. 2011;12:489-95.

73. Iwata Y, Suzuki N, Ohtake H, Kamauchi S, Hashimoto N, Kiyono $\mathrm{T}$, et al. Cancer cachexia causes skeletal muscle damage via transient receptor potential vanilloid 2-independent mechanisms, unlike muscular dystrophy. J Cachexia Sarcopenia Muscle. 2016;7:366-76.

74. Ferrer-Montiel A, Fernandez-Carvajal A, Planells-Cases R, Fernandez-Ballester G, Gonzalez-Ros JM, Messeguer A, et al. Advances in modulating thermosensory TRP channels. Expert Opin Ther Pat. 2012;22:999-1017.

75. Katanosaka K, Takatsu S, Mizumura K, Naruse K, Katanosaka Y. TRPV2 is required for mechanical nociception and the stretchevoked response of primary sensory neurons. Sci Rep. 2018;8:16782.

76. Muller C, Morales P, Reggio PH. Cannabinoid ligands targeting TRP channels. Front Mol Neurosci. 2019;11:487.

77. Liu D, Song L, Dai Z, Guan H, Kang H, Zhang Y, et al. MiR-429 suppresses neurotrophin-3 to alleviate perineural invasion of pancreatic cancer. Biochem Biophys Res Commun. 2018;505:1077-83.

78. Yamamoto K, Tanaka S, Fuseya S, Ishida T, Zhang H, Kawamata $\mathrm{T}$, et al. Knockdown of TRPV2 channels in sensory neurons increases limb use and weight bearing but does not affect spontaneous flinching behavior in a mouse model of bone cancer. Mol Pain. 2018;14:174480691881994. 